\title{
Key Technologies and Alogrithms' Application in Agricultural Food Supply Chain Tracking System in E-commerce
}

\author{
Lijuan Huang ${ }^{1}$ and Pan $\mathrm{Liu}^{2}$ \\ ${ }^{1}$ Guangzhou University, Guangzhou 510006, Guangdong, China \\ ${ }^{2}$ Shanghai Business School, Shanghai 201400, China \\ huanglijuan66s@126.com, pan1008@163.com
}

\begin{abstract}
In nowadays' world, frequently occurred agricultural food safety events have severely done harm to people's health and directly initiated great trust crisis to governments and agricultural food enterprises, and the Ecommerce as virtual economy can enlarge the trust crisis terrifically. As a result, to establish unified traceability information sharing platform of agricultural supply chain has become an urgent task for the related organizations. This paper begins with an exploratory research on design and realization of Agricultural Food Supply Chain Tracking System (AFSCTS) in E-commerce environment, which can provide a powerful technical support for timely tracing and finding the link where a agricultural food safety problem happens in the supply chain. This paper has 5 sections: the first to briefly introduce the concepts relevant to AFSCTS and the significance of this research; the second to analyze three key technologies (RFID technology, database integration technology and data security technology) utilized in AFSCTS; the third to elaborate on two key algorithms (traceability algorithm and data encryption algorithm) designed in AFSCTS; the fourth to design and implement AFSCTS based on three key technologies and two key algorithms, and the overall system frame and function structure are narrated detailedly in the paper; the last to apply the AFSCTS in Nanfeng county (the hometown of famous Nanfeng Orange in China) for tracing agricultural food, and the system implementation has achieved experimental success.
\end{abstract}

Keywords: agricultural food, tracking system, supply chain, E-commerce.

\section{$1 \quad$ Introduction}

\subsection{Related Concepts}

Currently, there is no a unified authority definition of Food Supply Chain Tracking System (FSCTS). According to ISO standards (ISO22005, 2013), tracking system can identificate and track object position change by recording items code. Food traceability can be defined as tracking all stages (from production, processing to sales) about food products (J. A. Monahan, 2012; T. Simpson, 2013) [1]. The European 
commission defines that FSCTS is the Management Information System (MIS) to trace material used in animals or feeds in any specified stages of food supply chain(M. Reynolds, 2012; R. Angeles 2012) [2-3]. The definition of Agricultural Food Supply Chain Tracking System (AFSCTS), provided by the United Nations Codex Alimentations Commission (UNCAC), is a computer system to trace agricultural food in production, processing, distribution and sale process of agricultural supply chain (F. Fisher, 2013) [4].

In western nations, more and more attention is focused on the research of Tracking System, such as Tracking System based on products supply chain (May Tajima, 2013), Tracking System based on hospital applications (T. Monahan, 2012; P. Stanfield, 2013), and children's tracking system for large amusement parks (Xiaodong Lin, etc., 2012) [5-7]. These systems have achieved traceability function by database technology and RFID technology. With continuous development of RFID technology and continuous decline of its cost, research on Tracking System is getting increasing attention, especially in monitoring the quality and safety of food (MinBo Li, ZhuXu Jing, Chen Chen, 2012) [8]. These applications based on RFID have accelerated RFID technology development in E-commerce environment (Feng Huang, Peng Hao, HuaRui Wu, 2012) [9].

European Bovine Spongiform Encephalopathy (BSE) crisis in EU countries represented as the global scope vicious food safety incidents' outbreak. CAC Biotechnology Intergovernmental Task Force Meeting divided FSCTS into five parts (records management, inquiring management, marking management, liability management and credit management). According to the features differences of agricultural food Tracking System, Elise Golan, an American scholar, sets three standards to measure AFSCTS: breadth, depth and precision (X. L. Kwan, 2010) [10]. Among them, breadth means the information scope that the system contained, depth means the distance that traceability information goes forward or backward, and precision means the ability that can determine unsafe food source or certain features of goods (Elise Golan, 2008) [11].

Generally, AFSCTS is divided into two kinds: one is oriented to the consumers, this means tracing from the upstream node of food supply chain to downstream node (e.g. consumers); the other is oriented to the suppliers, this means tracing from the downstream node of food supply chain to the upstream node (e.g. food production source). In view of the importance of food safety, there are more researches focused on the latter tracking system in E-commerce environment.

\subsection{Background and Significance}

In recent years, frequently occured food safety events (such as SanLu milk powder, Foot-and-mouth disease, Avian flu, PRRS, etc) have been severely doing harm to consumers' health, causing huge economic losses in many countries, and directly initiating great trust crisis to governments and enterprises in food supply chain (N. K. Porter, 2011). In order to help the governments and the related organizations timely find food safety problems in the process of production or circulation and make the responsibility of enterprises or related departments clear, it has become an urgent and important task for domestic and foreign governments and the related organizations to establish unified traceability information sharing platform in food supply chain. 
Developed countries have issued a series of relevant laws and regulations, which require that all enterprises engaged in production, processing, packaging or managing food of human or animal must provide traceability information platform (C.W. Lau, 2011). In the United States, food enterprises must trace and find food problem within 48 hours, or face large sum of astonishing fines; In Japan, only through many hurdles, then food could be on dining-table; In France, if a store is found to sell expired food, the store will be shut; In Canada, 80 percent of food can be traced to source, and the country is realizing "the brand Canada" strategy (Stanford, 2010).

At present, China's frequent malignant events of food safely (such as toxic powder event, Sudan red event, turbot fish event, and bonny big event) directly shocks the Chinese Central government. Food safety problem has become the focus of attention of the whole China society (C. J. Tao, 2011). Researching and developing AFSCTS is imminent in China, and it can provide powerful technical support to crack down on food crime and safeguard people's health. In this paper, we planned and designed AFSCTS based on three key technologies and two key alogrithms.

\section{Key Technologies}

There are three key technologies (RFID technology, database integration technology and data security technology) utilized in AFSCTS under the environment of E-commerce.

\subsection{RFID Technology}

RFID (Radio Frequency Identification), arises in recent years, is a kind of automatic identification technology. It has become one of indispensable support technologies in Internet of Things today. RFID has some characteristics such as unique identifier, fast read and write, untouched identification, mobile identification, and multi-target recognition (1000 tags can be simultaneously identified per second) make it possible to realize efficient traceability for supply chain system. In RFID System, digital memory chips with unique electronic commodity codes can be pasted on single food product, and receiving equipment can activate RFID tags, read and change data, and transmit data to host computer for further processing the data. Operating principle of RFID technology is shown in Fig.1.

At present, the method based on traditional bar codes can not realize traceability management for the whole food supply chain. Compared with bar code technology, RFID tags are more suitable for managing the whole process of food supply chain from farmland to dining-table. Because there are unique identification codes, data erase duplication, tag with large storage for data, and fast response for identifying tag with a long service life, RFID can be used in bad condition such as high temperature and humidity. Applying RFID technology can not only identify every unit of goods but also may effectively identify each node of supply chain to track and trace each link of supply chain. These links include processing, packaging, storage, transportation and sales. This may timely find out existing problems and properly handle them. 


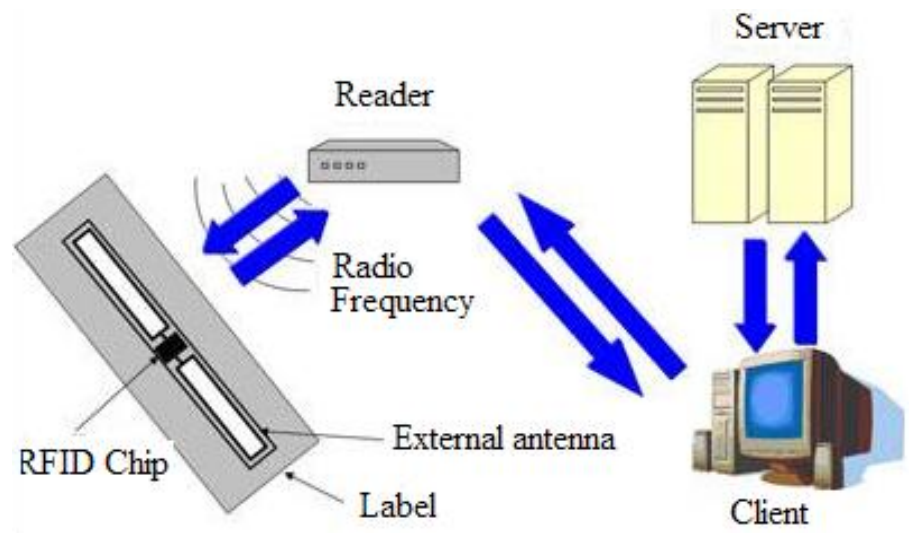

Fig. 1. Operating principle of RFID technology

\subsection{Database Integration Technology}

Heterogeneous database integration technology can merge and share data resources or hardware resources from different databases. Through database integration technology, information of monitoring product from databases of each enterprise in supply chain will be integrated. This can achieve to trace food in whole supply chain, and each enterprise can use its database without redesigning or rebuilding new databases. This can save costs for tracing food supply chain.

In recent years, technologies applied to integration of databases mainly include middle ware, mobile agent and XML. The features of mobile agent is autonomy, mobility and collaboration. There are also distributed self-learning and self-reasoning about mobile Agent. So, the rapid development of mobile agent can give a new way for research on the heterogeneous database integration technology. XML has gradually become industry standard of data representation and information exchange, and it may provide a platform for transferring data of relational databases of heterogeneous and different platforms. The combination with mobile agent and XML could improve the efficiency of user queries in process of heterogeneous database integration. XML is a model used to describe data structure. It utilizes a mechanism to describe contents of related table in database and standardized combination form in files. XML also defines description rules such as file structure, data type and so on. XML has been widely used to access to heterogeneous data sources and transmission of middle ware. As network technology and software technology continue to evolve, distributed and heterogeneous topology has become remarkable characteristics of all kinds of calculation and development environment of system. Heterogeneous database integration technology has been considered to be hotpot and difficult issues in Database fields by domestic and foreign academia and industry.

\section{$3 \quad$ Key Algorithms}

There are two key algorithms (Traceability algorithm and data encryption algorithm) designed in AFSCTS under the environment of E-commerce. 


\subsection{Traceability Algorithm}

In China, the traditional traceability algorithm can only trace a enterprise but can not trace all node in the supply chain, and the traceability procedure only starts after unsafe food have done harm to the society. As a result, this traceability is often too late and not so successful.

In order to establish tracking and tracing model in Tracking System, we need to make sure the EPC coding about the products in the supply chain and the mapping of the data information in Tracking System. In the actual application system, mainly information of product coding contains two parts: identification information and record information. The identification information can be used to set the only logo to the product, and it gets the EPC logo through multi-level to make sure the continuity of tracing process and tracking process. Generally, the record information contains main information of the product. It mainly is used by supply chain enterprises to communicate between internal and external. The tracking information of product comes from the object events, and it changes tracing event information into tracing unit information for realizing tracing each unit back on the supply chain.

Product traceability unit can be defined as formula (5):

$$
S_{i}=\left\{s_{1}, s_{2}, \cdots, s_{n}\right\}
$$

$S_{\mathrm{i}}$ is used to describe object products set in the supply chain, and it also consists other objects in same supply chain. The change of each object is corresponding to the corresponding events. So each object $E_{i j}$ contains event set eik, and $k \in\{1,2, \ldots, \mathrm{j}\} . E_{i j}$ can be expressed as formula (6):

$$
E_{i j}=\left\{e_{i 1}, e_{i 2}, \cdots, e_{i j}\right\}
$$

Where, object $i$ contains events $j$. Each event is corresponding to the change of information, such as product status, position and properties, and tracing events can effectively ensure continuity of traceability. Therefore, Object $\mathrm{Si}$ in time $\mathrm{t}$ runs through a supply chain node, and then its status $\mathrm{Ct}(\mathrm{Si})$ can be described by several parameters as formula (7):

$$
C_{t}\left(S_{i}\right)=\left\{M, E, t, I D_{t}, \text { Type }_{t}\left(S_{i}\right), \operatorname{Loc}\left(S_{i}\right), \operatorname{Step}\left(S_{i}\right), \operatorname{Real}_{t}\left(S_{i}\right)\right\}
$$

Where, Time $t$ is used to express the status of time utility.

ID $t$ is the corresponding labels about the object $\mathrm{S} i$ at moment $t$.

Typet $(\mathrm{S} i)$ is the business operation types in the process of the supply chain about the object $\mathrm{S} i$ at moment $\mathrm{t}$.

$\operatorname{Loc}(\mathrm{S} i)$ is the position in the process of supply chain about the $\mathrm{S} i$ at moment $\mathrm{t}$.

$\operatorname{Step}(\mathrm{S} i)$ is the business step at supply chain node about $\mathrm{Si}$ at moment $t$.

$\operatorname{Realt}(\mathrm{Sj})$ is used to describe the association contact between object $\mathrm{Si}$ and other object $\mathrm{Sj}$, such as the relationship between the product object and the packing bag object, and it realizes the binding and split between different objects.

Object value $\mathrm{M}$ is serial number about the object $\mathrm{Si}$ associated with the other object $S j$. M can be expressed as formula (8), where $i$ is not equal to $j$ : 


$$
M=\sum_{j=1}^{n} \operatorname{Mat}_{t}\left(S_{i}\right)
$$

Event value $E$ is sum of all Function $E j$ with parameter $S i$ in given time $t$, and it can be computed as formula (9):

$$
E=\sum_{j}^{n} E_{j}\left(S_{i}\right)
$$

AFSCTS designed in this paper can not only trace a node, but also trace the whole supply chain. It can not only support traceability after the event, but also support traceability before the event. The AFSCTS with real-time monitoring can eliminate the potential unsafely of food and to protect people's life safety. Traceability algorithm can obtain and express corresponding tracing results through EPCIS interfaces. Pseudo-code of main tracing algorithm in the AFSCTS is described as follows :

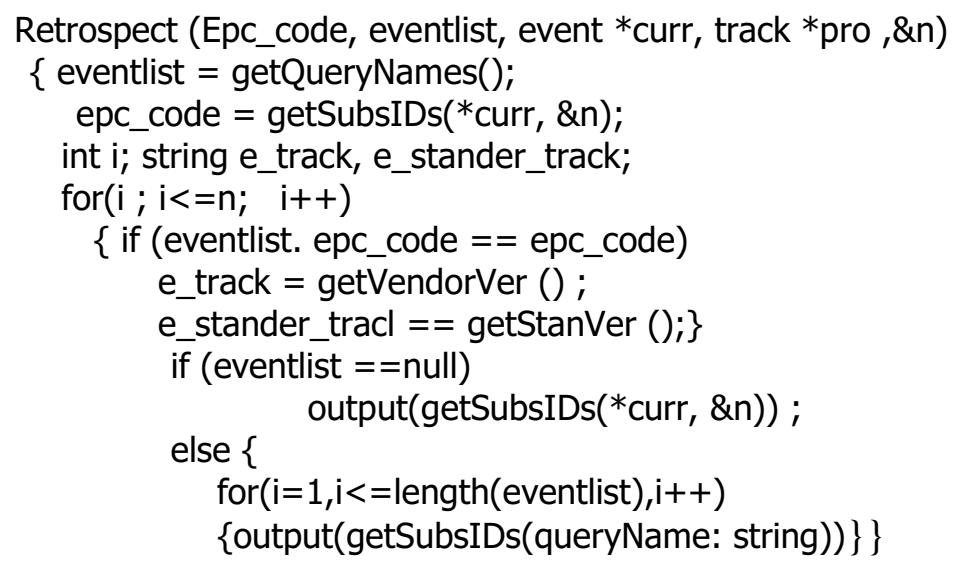

The main interfaces of EPCIS in the ASCTS have been specified in Table I.

Table 1. The main interfaces parameter of EPCIS

\begin{tabular}{cccc}
\hline No. & Name & Parameter & Type \\
\hline 1 & Subscribe & Query Name & string \\
2 & Subscription Controls & Subscription_ ID & string \\
3 & unsubscribe & Subscription_ID & string \\
4 & getQueryNames & & list \\
5 & getVendorVersion & string \\
6 & getVendorVersion & string \\
\hline
\end{tabular}




\subsection{Encryption Algorithm}

There are some important information ( e.g. money or assets information and consumer information ) in AFSCTS. It is hard for enterprise to define potential intruders, because information resources are sharing by all firms in supply chain. If food supply chain information system is illegally invaded, that may cause huge financial losses for the firm which is invaded, or even all node enterprises in the whole supply chain may suffer huge losses. Data encryption is very important for the system because integrated database is used in the system. It is essential to use electronic tag with identity authentication and encryption system in order to protect commercial secrets of each node enterprise in AFSCTS. At present, the research about data security of RFID is paid more attention. Advanced Encryption Standard (AES) is post by NIST in 1997, and now it is considered to be a replacement for DES that represents traditional symmetric encryption algorithms. After several years, NIST sifts many algorithms and eventually determines to take Rijndael algorithms as AES, which is invented by Vincent Rijmen and Joan Daemen as the replacement of DES. NIST have established a new standard of AES in May, 2002. Rijndael uses a very limited storage of RAM and ROM, this makes Rijndael to be a excellent candidate when it is applied in resource-constrained environment. Therefore, it is possible to realize encryption of RIFD technology. So this research proposes that AES algorithm is transported to the food supply chain Tracking System for guaranteeing data security in shared database. But taking into account such factors as the cost of technology, successfully applying Rijndael in RFID needs to be studied; this article raised the idea of the data encryption.

Main part of the Rijndael is Mix Column transform, and it has mainly accessed knowledge of polynomial arithmetic. Here are the domain F2n of arithmetic, which can be calculated as formula (10) :

$$
f(x)=a_{n-1} x^{n-1}+a_{n-2} x^{n-2}+\cdots+a_{1} x+a_{0}
$$

Where, coefficient $a_{i}$ is from Polynomial coefficients as the $n$-tuple, and the tuple can correspond to the number of binary computer. So, it is convenient to realize program to apply this algorithm in computer. Rijndael algorithm includes: $S$-box, shiftRow transform, MixColumn transform, and AddRoundKey. AddRoundKey is regarded as the last step, and each round key is combined with the message by XOR operation, which may have formula (11) to represent, and $j=0, \ldots, L_{b}-1$ :

$$
\left(b_{0, j}, b_{1, j}, b_{2, j}, b_{3, j}\right) \leftrightarrow\left(b_{0, j}, b_{1, j}, b_{2, j}, b_{3, j}\right) \oplus\left(k_{0, j}, k_{1, j}, k_{2, j}, k_{3, j}\right)
$$

AES uses a loop structure to realize iterative encryption and input data by permutations and substitutions. Permutations are rearranging data. Substitutions are replacing another with a unit of data. ASE uses all kind of different technologies to achieve permutations and substitutions. In our AFSCTS, the key can be produced by data encryption algorithm. The pseudo-code is as follows: 


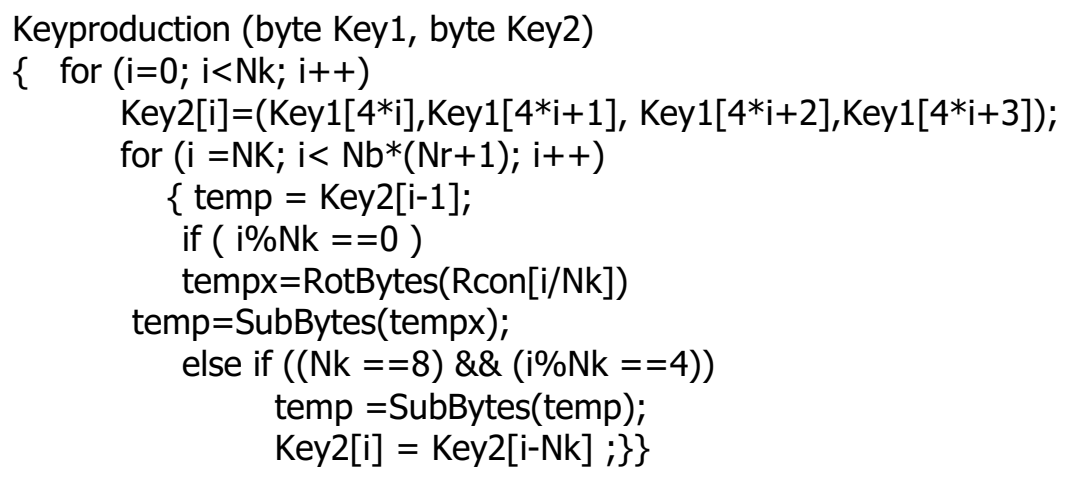

\subsection{Data Security Algorithm}

With rapid development of network technology, the degree of openness, sharing and interconnection of Internet are continuously improved. It is more and more important for information safety to construct AFSCTS, which should take reliable measures to guarantee data safety. At present, there are several methods of security design about AFSCTS based on RFID technology.

(1) Data of RFID tags is encrypted. EPC code is encrypted by RSA algorithm and then the code is written down in tags. Literacy device with private-key read labels cipher text can encode and decrypt to get EPC gold-digging. RSA algorithm is the evolution of RICE which was published by Ron Rivest, Adi Shamir and Len Adleman from MIT in 1987. RSA is a kind of block cipher. The text passwords and cipher text of the RSA are integer between 0 and $\mathrm{n}-1$. Based on block $\mathrm{M}$ of text passwords and block $\mathrm{C}$ of cipher text, the process of encryption and decryption could be described as formula (1) and formula (2):

$$
\begin{gathered}
C=M^{e} \\
M=C^{d} \bmod n=M^{e . d} \bmod n
\end{gathered}
$$

Based on the above formulas, both sender and receiver have known the variable $\mathrm{n}$ and variable e, so the only requirement is that receiver has to know the variable $d$. The equation of public key is $P U=\{e, n\}$, and the equation of public key is $P R=\{d, n\}$.

(2) RFID reader approves the authenticity of labels by HB Protocol, and HB Protocol is introduced by Hoper and Blum (2012). HB Protocol can be used to solve personal identity authentication, and it can also effectively solve message of asking for reply in process of eavesdropping and authentication.

(3) Though database decipherments to protect the security of system data. During the process of encrypting AFSCTS data, encrypted data have been stored in the server. The data in the server is safe, because there are not keys on the server. Even if someone can make an incursion to the server, and he has no access to the data encrypted. The client system has a copy of encryption key, so users can obtain the data through corresponding method. During the process of encrypting AFSCTS data, each record of the database is encrypted by block. Each line named Ri can be taken as 
a continuous block which can be expressed as formula (3) and the entire line can be expressed as formula (4):

$$
\begin{aligned}
& B_{i}=\left(x_{i 1}\left\|x_{i 2}\right\| \ldots \| x_{i M}\right) \\
& E\left(k, B_{j}\right)=E\left(k,\left(x_{i 1}\left\|x_{i 2}\right\| \ldots \| x_{i M}\right)\right)
\end{aligned}
$$

(4) Using CA technology can realize RFID data safety in the process of transmission on the net. User's identity is identified between RFID client and data center in tracking server rooms by digital signature technology. In the process of transmission, message authentication code is added into confidential RFID data so as to realize packet encryption and data integrity checks.

According to safety requirements of AFSCTS, we can independently or collectively apply those safety technologies. Eclectically considering between cost investment and security degree, we realized storage and encryption algorithms of security system by software. Certainly, they can rely on hardware to achieve, but with lower processing power and higher cost of tags encrypted than the former.

\section{Application and Realization}

At present, AFSCTS is designed and applied in Nanfeng county. Nanfeng county is located in the southeast of Jiangxi Province, China. It is famous orange hometown, where Nanfeng Orange was regarded as "Orange King" by the president of former Soviet Union Stalin and also praised as "Gold Orange" by Chinese Premier Wen Jiabao.

Beautiful Nanfeng is abundant not only in orange but also in other characteristic agricultural foods (e.g. rice, pickles, turtle, bean milk skin and beating tea). Although international market demand is very large for these agricultural foods, actual exports cannot meet them, even Nanfeng Oranges was unsalable in domestic market at one time, partly because there is no tracking system of agricultural foods, so it is very difficult to track agricultural foods and to find the reasons when food unsafely events happen. Some unsafely events occurred in recent years have seriously done harm to the world brand of Nanfeng Orange and shares of international market. So, to ensure quality safety of agricultural foods is very meanful work for striving to expand the international market. Based on the above three key technologies and two key algorithms, overall framework and function structure of Nanfeng county's AFSCTS are described as follows.

\subsection{Overall Framework}

General idea of the system design is described as follows: Integrate the data processed by Event handler, then store the above data in a public database of AFSCTS. Because the integrated data usually involves business secrets, supply chain enterprises can encrypt the data for protecting business secrets. The user who has permission can access public database to query the corresponding data. Integrated database involves the whole supply chain of each enterprise's monitoring data, so there is huge amount 
of data in this shared database, and it needs to store the previous data regularly in historical database.

When a tracing request involves historical data, it can use tracing query by calling the historical database or archiving the data in the historical database to the current data. Integration database of AFSCTS generally stores each enterprise's monitoring data, and it adds a historical database for relieving the pressure of integration database and increasing query sensitivity of current product status information. Based on supply chain integration database, we can design a special food product traceability platform conveniently for the users who have permissions to trace products, and feedback the result of retroactive to users directly. Here the user usually refers to the customer who has key, because it involves the data security and the data on all aspects of the product may be encrypted. The AFSCTS for Nanfeng county can also track the flow of products, and it's more convenient for enterprises to analyzing the market.

Overall framework of Nanfeng county's AFSCTS is shown in fig.2, where this framework includes RFID data flow based on complex event handling mechanism of EPCIS. According to the EPCIS standards, AFSCTS can be divided into two parts (Master data and Event data), and the two events include simple events and complex events. According to the business function of complex events, it can be divided into Object Event, Quantity Event, Transaction Event and Aggregation Event. Filtering repeated data and classifying into various incidents based on its function, EPCIS has special events capture operation module, and it will capture events to handle the

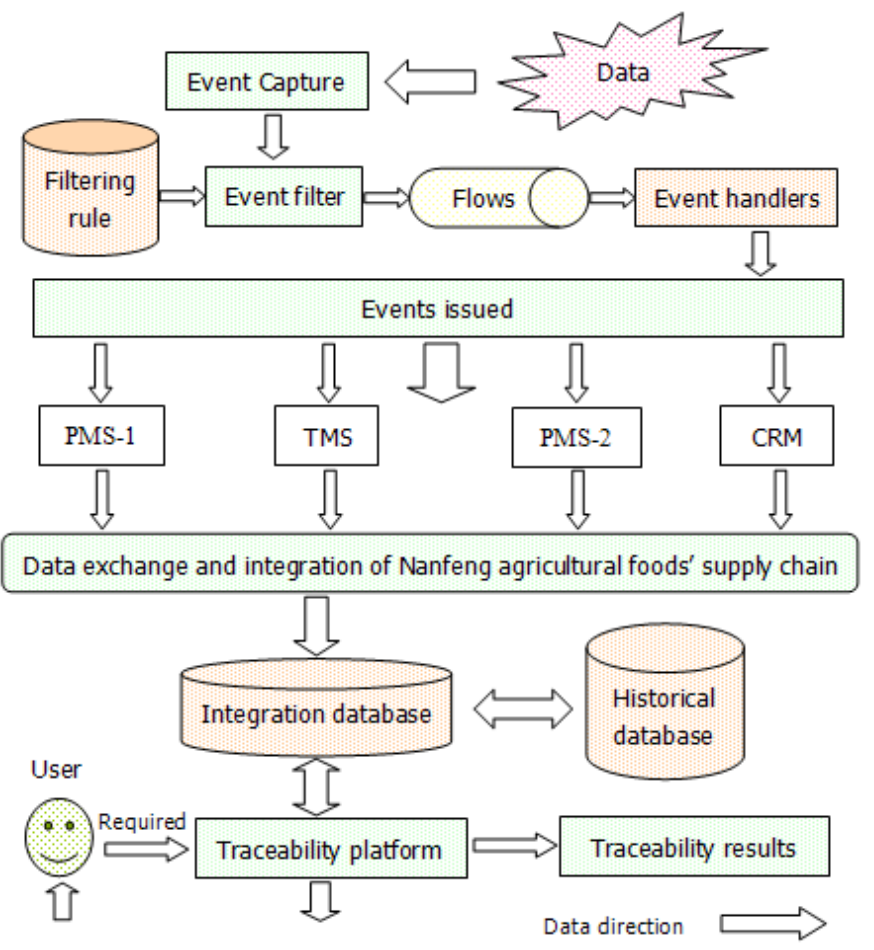

Fig. 2. Overall framework of Nanfeng county's AFSCTS 
processing center in the stream of event, then issue these events. The capture interface can be defined as Capture (event:List< EPCISEvent $>$ ): void.In the whole of this operation, data is employed by RFID technology, then deposited in each link database of the subsystems in AFSCTS respectively.

\subsection{Function Structure}

Based on the above design thought, monitoring information of products in the whole Nanfeng agricultural food supply chain is integrated, and then stored in integration database of AFSCTS. Hence, any authorized nodes in the supply chain (e.g. enterprises, Governments, or consumer) can trace the sources and whereabouts of Nanfeng agricultural foods conveniently from this database. In this system, it's worth saying that Nanfeng agricultural foods can be reversely tracked and positively tracked in the supply chain.

There are main four functions of AFSCTS to be designed for Nanfeng agricultural food supply chain in the paper, namely planting monitoring, processing monitoring, transportation monitoring and sales monitoring. Its function structure is shown in Fig. 3.

(1) Planting monitoring: through RFID technology, this system mainly real-timely records the detail of Nanfeng agricultural products' planting information, such as plant varieties, seeding records, irrigation records, fertilization records, pest control records, and manage the information about pesticide purchase, storage, use and safety period. If pesticide expired or banned pesticide appears, it will give off warning information. After Nanfeng agricultural foods are picked, picking date, plot numbers and picking sequence will be batched by the times number. Put all basic information including management personnel's name together in a label card written by the times number, thus make each participating object on tracing chain of agricultural foods.

(2) Processing monitoring: because information about Nanfeng agricultural foods can be easily added to labels, once these foods enter the processing link, the related processing enterprise can read the label information of the products first, and then according to the needs in itself, change or add the corresponding information (such as processing enterprise name, processing time, processing address and packed weight) to the labels.

(3) Transportation monitoring: when preparing Nanfeng agricultural foods for loading, it uses fixed literacy to detect the products in loading area. AFSCTS first reads the label card of transport vehicles automatically, this label card records basic information of the vehicles such as nameplate and owner'name. If the label card is read with illegal data, it will alarm automatically. After loading finished, loading personnel needs to add loading information to label card, such as loading time, start time, destination and so on.

(4) Sales monitoring: Manager can read label by reader automatically to establish special sales monitoring system (such as monitoring system of supermarkets) based on RFID technology. If the customers pay online, AFSCTS can not only trace information record of this customer and know final whereabouts of Nanfeng agricultural foods, but also it can trace these products sourcing in the upper supply chain. 


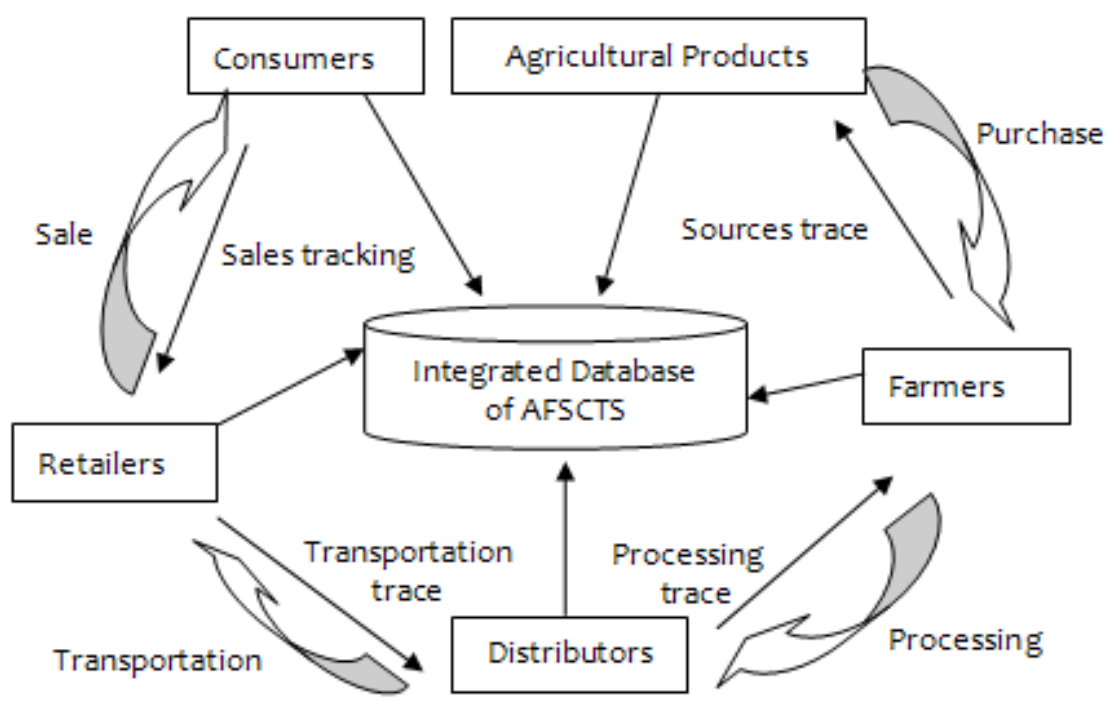

Fig. 3. Flow-process diagram of AFSCTS

\section{Conclusion}

There is an old saying "Food is the paramount necessity of the people." In recent years, frequently occurred agricultural food safety events all over the world have been severely doing harm to people's health, and also directly initiating great trust crisis to governments and the society. In order to timely trace and find unsafe agricultural food source and provide a powerful technical support to crack down on food crime and safeguard people's health, designing and developing AFSCTS is of great practical value and social significance and is supported by the government and all walks of life. Based on three key technologies and two key alogrithms, our research groups begins with exploratory research for designing and planning an AFSCTS in E-commerce environment, which is applied in Nanfeng county (the hometown of famous Nanfeng Orange in China) for tracing food safety of Nanfeng agricultural Supply Chain, and the experimental result of this system implementation is successful. As supply chain Tracking System has seldom been applied to agricultural food supply chain in China, the research and application is an innovation, and its fruit can be used in pharmaceutical and dangerous goods industry as well as agricultural food supply chain.

Acknowledgment. The authors thank the anonymous reviewers for their valuable remarks and comments. This work is supported by Shanghai Natural Science Fund (No.13ZR1429600), 2012 Teaching reform Project of Colleges and Universities of Jiangxi Province (Grant No. JXJG-12-3-16), the 11th Five-year Social Science Planning Project of Jiangxi Province in 2010 (Grant No. 10GL35), Social Science 
Research Project of Guangzhou University, Innovation Program of Shanghai Municipal Education Commission (No. 13YZ141), and Young teacher training scheme of Shanghai Universities (No. SXY12014).

\section{References}

1. Tajima, M.: Value of RFID in supply chain management. Journal of Purchasing \& Supply Management 69(12), 261-273 (2013)

2. Kshetri, N.: Barriers to e-commerce and competitive business models in developing countries: A case study. Electronic Commerce Research and Applications 6(4), 443-452 (2013)

3. Angeles, R.: Supply-Chain Applications and Implementation Issue. Information Systems Management 23(10), 51-65 (2012)

4. Nath, B., Reynolds, F.: Want R, RFID technology and applications. IEEE Pervasive Computing 5(1), 22-24 (2012)

5. Knospe, H., Pohl, H.: Information Security Technical Report. RFID security 9(4), 39-50 (2012)

6. Ko, J.M., Kwak, C., Cho, Y., Kim, C.O.: Adaptive product tracking in RFID-enabled large-scale supply chain. Expert Systems with Applications 38(3), 1583-1590 (2012)

7. Fisher, J.A., Monahan, T.: Tracking the social dimensions of RFID systems in hospitals. International Journal of Medical Informatics 77(3), 176-183 (2013)

8. Huang Li-juan, A.: personalized recommendation system for e-supply chain based on improved fat-growth algorithm. Journal of Applied Mathematics and Statistics 45(13), 135-141 (2013)

9. Simpson, L.K.T., Stanfield, P.: A model for quantifying the value of RFID-enabled equipment tracking in hospitals. Advanced Engineering Informatics 25(8), 23-31 (2013)

10. Jing, M.X., Chen, C.: The application of RFID in tracing system. Computer Integrated Manufacturing Systems 126(10), 241-253 (2012)

11. Huang, F., Hao, P., Wu, H.R.: Application of RFID middleware in food product safety Tracking System. Transactions of the CSAE 24(5), 177-181 (2012)

12. Wang, F.S., Liu, S.R., Liu, P.Y.: A temporal RFID data model for querying physical objects. Pervasive and Mobile Computing 6(3), 382-397 (2012)

13. Huang, L., Yu, P., Luo, Q., Zou, C.: E-Tourism Supply Chain Evaluation Based On Ahp And Fce Method. Journal of Theoretical and Applied Information Technology 45(2), 702-709 (2012)

14. Hopper, N.J., Blum, M.: Secure Human Identification Protocols. In: Boyd, C. (ed.) ASIACRYPT 2001. LNCS, vol. 2248, pp. 52-66. Springer, Heidelberg (2001)

15. Lee, C.K.M., William, H., Ho, G.T.S., Lau, H.C.W.: Design and development of logistics workflow systems for demand management with RFID. Expert Systems with Applications 38(5), 428-437 (2011)

16. Saidi, K.S., Teizer, J., Franaszek, M., Lytle, A.M.: Static and dynamic performance evaluation of a commercially-available ultra wideband tracking system. Automation in Construction 29(3), 453-458 (2013) 